\title{
CONSTRUCTING CERTAIN SPECIAL ANALYTIC GALOIS EXTENSIONS
}

\author{
ANWESH RAY
}

\begin{abstract}
For every prime $p \geq 5$ for which a certain condition on the class group $\operatorname{Cl}\left(\mathbb{Q}\left(\mu_{p}\right)\right)$ is satisfied, we construct a $p$-adic analytic Galois extension of the infinite cyclotomic extension $\mathbb{Q}\left(\mu_{p^{\infty}}\right)$ with some special ramification properties. In greater detail, this extension is unramified at primes above $p$ and tamely ramified above finitely many rational primes and its Galois group over $\mathbb{Q}\left(\mu_{p} \infty\right)$ is isomorphic to a finite index subgroup of $\mathrm{SL}_{2}\left(\mathbb{Z}_{p}\right)$ which contains the principal congruence subgroup. For the primes 107, 139, 271 and 379 such extensions were first constructed by Ohtani and Blondeau. The strategy for producing these special extensions at an abundant number of primes is through lifting two-dimensional reducible Galois representations which are diagonal when restricted to $p$.
\end{abstract}

\section{Introduction}

Let $p$ be a prime number, the tame Fontaine-Mazur conjecture (conjecture 5a in [2]) posits that an infinite Galois extension of a number field whose Galois group over $\mathbb{Q}$ is isomorphic to a $p$-adic analytic group is either ramified at infinitely many primes or is infinitely ramified at a prime dividing $p$. It is natural to ask if such extensions exist once we pass up an infinite cyclotomic extension $\mathbb{Q}\left(\mu_{p^{\infty}}\right)$. We show that there are abundantly many primes $p$ for which there exists such an extension of $\mathbb{Q}\left(\mu_{p^{\infty}}\right)$ whose Galois group is isomorphic to a finite index subgroup of $\mathrm{SL}_{2}\left(\mathbb{Z}_{p}\right)$ with tame ramification above finitely many rational primes and unramified at primes above $p$.

Let $p \geq 5$ be a prime and $\zeta_{p}=e^{\frac{2 \pi i}{p}}$. Denote by $H_{\mathbb{Q}\left(\mu_{p}\right)}^{\prime}$ the $p$-Hilbert Class field of $\mathbb{Q}\left(\mu_{p}\right)$, we identify $\operatorname{Gal}\left(H_{\mathbb{Q}\left(\mu_{p}\right)}^{\prime} / \mathbb{Q}\left(\mu_{p}\right)\right)$ with the $p$-part of the class group $\operatorname{Cl}\left(\mathbb{Q}\left(\mu_{p}\right)\right)$.

We associate a Galois representation to class group data. Denote by

$$
\mathcal{C}:=\operatorname{Cl}\left(\mathbb{Q}\left(\mu_{p}\right)\right) \otimes \mathbb{F}_{p}
$$

and $\bar{\chi}$ the $\bmod p$ cyclotomic character. The Galois module $\mathcal{C}$ decomposes into isotypic components

$$
\mathcal{C}=\bigoplus_{i=0}^{p-2} \mathcal{C}\left(\bar{\chi}^{i}\right)
$$

where

$$
\mathcal{C}\left(\bar{\chi}^{i}\right)=\left\{x \in \mathcal{C} \mid g \cdot x=\bar{\chi}^{i}(g) x \text { for all } g \in \operatorname{Gal}\left(\mathbb{Q}\left(\mu_{p}\right) / \mathbb{Q}\right)\right\} .
$$

By class field theory $\mathbb{F}_{p}$-line in $\mathcal{C}\left(\bar{\chi}^{i}\right)$ gives rise to an extension $L / \mathbb{Q}$ contained in the $p$ Hilbert Class field $H_{\mathbb{Q}\left(\mu_{p}\right)}^{\prime}$. Since such a line is Galois stable, the extension $L$ is Galois over $\mathbb{Q}$. Any choice of isomorphism $\operatorname{Gal}\left(L / \mathbb{Q}\left(\mu_{p}\right)\right) \stackrel{\sim}{\rightarrow} \mathbb{F}_{p}$ gives rise to an element

$$
\beta \in H^{1}\left(G_{\mathbb{Q}}, \mathbb{F}_{p}\left(\bar{\chi}^{i}\right)\right) \simeq \operatorname{Hom}\left(G_{\mathbb{Q}\left(\mu_{p}\right)}, \mathbb{F}_{p}\left(\bar{\chi}^{i}\right)\right)^{\operatorname{Gal}\left(\mathbb{Q}\left(\mu_{p}\right) / \mathbb{Q}\right)} .
$$

This class does not depend on the choice of isomorphism. The class $\beta$ coincides with a reducible Galois representation

$$
\bar{\rho}: G_{\mathbb{Q}} \rightarrow \mathrm{GL}_{2}\left(\mathbb{F}_{p}\right)
$$


defined by $\bar{\rho}=\left(\begin{array}{cc}\bar{\chi}^{i} & \beta \\ 0 & 1\end{array}\right)$. The representation satisfies a number of conditions:

(1) $\bar{\rho}_{\left\lceil G_{\mathbb{Q}\left(\mu_{p}\right)}\right.}$ is indecomposable,

(2) $\bar{\rho}_{\left\lceil G_{\mathbb{Q}\left(\mu_{p}\right)}\right.}=\left(\begin{array}{ll}1 & \beta \\ 0 & 1\end{array}\right)$ is unramified at every prime,

(3) the local Galois representation at $p$ splits into a sum of characters $\bar{\rho}_{\left\lceil G_{p}\right.} \simeq\left(\begin{array}{cc}\bar{\chi}_{\left\lceil G_{p}\right.}^{i} & 0 \\ 0 & 1\end{array}\right)$.

The extension $L \neq \mathbb{Q}\left(\mu_{p}\right)$ and thus the cohomology class $\beta$ is non-trivial, from which condition 1 follows. As $L$ is contained in the Hilbert class field of $\mathbb{Q}\left(\mu_{p}\right)$ every prime of $\mathbb{Q}\left(\mu_{p}\right)$ is unramified in $L$. Condition 2 follows from this. By class field theory the principal prime-ideal $\left(1-\zeta_{p}\right)$ is split in the Hilbert class field of $\mathbb{Q}\left(\mu_{p}\right)$ and thus in $L$. Let $E$ denote the completion of $\mathbb{Q}\left(\mu_{p}\right)$ at $\left(1-\zeta_{p}\right)$. One deduces that $\beta_{\left\lceil G_{E}\right.} \in H^{1}\left(G_{E}, \mathbb{F}\left(\bar{\chi}^{i}\right)\right)$ is trivial. We observe that the order of $\mathrm{Gal}\left(E / \mathbb{Q}_{p}\right)$ is coprime to $p$. From a standard argument appealing to the vanishing of $H^{1}\left(\mathrm{Gal}\left(E / \mathbb{Q}_{p}\right), \mathbb{F}\left(\bar{\chi}^{i}\right)\right)$ and the inflation-restriction sequence it follows that $\beta_{\left\lceil G_{p}\right.}=0$. Condition 3 follows as a consequence.

For an introduction to the deformation theory of Galois representations, the reader may consult [4]. We examine deformations of $\bar{\rho}$ with some prescribed local properties. In particular, the local deformation condition at $p$ should ensure that on passing up the infinite cyclotomic extension $\mathbb{Q}\left(\mu_{p^{\infty}}\right)$, our deformations are unramified at primes $\mathfrak{p} \mid p$.

Our construction is based on that of Hamblen and Ramakrishna who in 3 deduce the weak form of Serre's conjecture for a reducible residual Galois representation which satisfies some further conditions. Their method is based on a local to global deformation theoretic argument. Implicit to this construction is a choice of a local deformation condition at each prime at which the residual representation is allowed to ramify. Deformations of the residual representation are to satisfy these local conditions. In particular, at $p$ there is a choice of a local deformation condition which satisfies some key properties, namely the deformation condition is liftable and balanced (see Definition 3.2). Hamblen and Ramakrishna prescribe the ordinary deformation condition (cf. [5 and [7) and we shall on the other hand be working with the diagonal deformation condition (see Definition 3.1). This deformation condition is liftable and balanced as we shall show (see Proposition 3.3). We shall then deduce be able to deduce the following theorem.

Theorem 1.1. Let $p \geq 5$ be a prime and $\mathcal{C}:=\operatorname{Cl}\left(\mathbb{Q}\left(\mu_{p}\right)\right) \otimes \mathbb{F}_{p}$. Suppose that there exists an odd integer $i \neq \frac{p-1}{2}$ such that $2 \leq i \leq p-3$ such that $\mathcal{C}\left(\bar{\chi}^{i}\right) \neq 0$. Let $\bar{\rho}=\left(\begin{array}{rr}\bar{\chi}^{i} & * \\ 0 & 1\end{array}\right)$ be the Galois representation associated to an $\mathbb{F}_{p}$-line in $\mathcal{C}\left(\bar{\chi}^{i}\right)$. There exist infinitely many lifts $\rho$ of $\bar{\rho}$

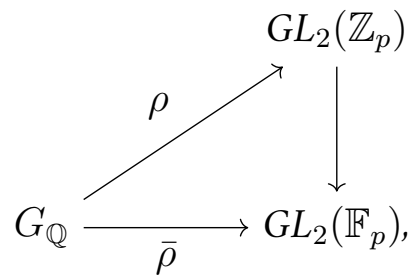

such that the following conditions are satisfied

- $\rho\left(G_{\mathbb{Q}\left(\mu_{p} \infty\right)}\right)$ contains the principal congruence subgroup of $S L_{2}\left(\mathbb{Z}_{p}\right)$

- the determinant of $\rho$ is $\chi^{i+p^{2}(p-1)}$

- $\rho_{\left\lceil G_{p}\right.}$ is a direct sum of characters $\rho_{\left\lceil G_{p}\right.}=\varphi_{1} \oplus \varphi_{2}$. In particular, $\rho$ is abelian at $I_{p}$.

- $\rho$ is unramified outside a finite set of primes. 
The lift $\rho$ gives rise to an extension $\mathbb{Q}(\rho)$ of $\mathbb{Q}\left(\mu_{p}\right)$ which is taken to be the fixed field of $\operatorname{ker} \rho \subset G_{\mathbb{Q}}$. This extension is unramified at primes above $p$. Furthermore, at a prime $l \neq p$, since the residual representation $\bar{\rho}$ is unramified, the lift $\rho$ is tamely ramified at all primes $l \in S /\{p\}$, we are left with the following result.

Corollary 1.2. Suppose that $p \geq 5$ be a prime and $i \neq \frac{p-1}{2}$ an odd integer between $2 \leq i \leq$ $p-3$ for which the isotypic space $\mathcal{C}\left(\bar{\chi}^{i}\right) \neq 0$ (cf. 1.1). There are infinitely many Galois extensions $L / \mathbb{Q}\left(\mu_{p^{\infty}}\right)$ for which

- the Galois group $\operatorname{Gal}\left(L / \mathbb{Q}\left(\mu_{p^{\infty}}\right)\right)$ topologically isomorphic to a subgroup of $S L_{2}\left(\mathbb{Z}_{p}\right)$ which contains the principal congruence subgroup.

- L is unramified at primes above $p$ and ramified above finitely many rational primes at which it is tamely ramified.

Proposition 1.3. The conclusion of Corollory 1.2 is satisfied at any prime $p$ such that

(1) $p \geq 5$,

(2) $p$ is irregular,

(3) $p \equiv 1 \bmod 4$,

(4) $p$ does not divide the class number of the totally real subfield $\mathbb{Q}\left(\mu_{p}\right)^{+} \subset \mathbb{Q}\left(\mu_{p}\right)$, i.e. Vandiver's conjecture is satisfied at $p$.

This seems to indicate that there are infinitely many primes at which $p$ the implication of Corollory 1.2 is satisfied.

Such $p$-adic extensions $L$ were first constructed by Ohtani $\mid 6$ and Blondeau [1] and their methods relied on lifting suitable irreducible Galois representations which are extraordinary at $p$. The construction in [6] and [1] relies on the existence of an eigenform $f$ with companion forms (and thus extraordinary at $p$ ) such the image of the residual representation $\bar{\rho}_{f}$ contains $\mathrm{SL}_{2}\left(\mathbb{F}_{p}\right)$. Computations for $p<3500$ show that there are precisely four primes $107,139,271$ and 379 for which such an eigenform $f$ exists.

Acknowledgements. I am very grateful to my advisor Ravi Ramakrishna for introducing me to his Galois-theoretic lifting method and for making helpful suggestions. I would also like to thank Christian Maire for many fruitful conversations. I would also like to thank the anonymous referee for some valuable suggestions.

\section{Summary of Notation}

- For all rational primes $v$ fix a choice of an algebraic closure of $\overline{\mathbb{Q}}_{v}$ and a choice of an embedding $\iota_{v}: \overline{\mathbb{Q}} \hookrightarrow \overline{\mathbb{Q}}_{v}$ extending $\mathbb{Q} \hookrightarrow \mathbb{Q}_{v}$. The absolute Galois group of $\mathbb{Q}_{v}$ is denoted by $G_{v}:=\operatorname{Gal}\left(\overline{\mathbb{Q}}_{v} / \mathbb{Q}_{v}\right)$. The embedding $\iota_{v}$ coincides with an inclusion $G_{v} \hookrightarrow G_{\mathbb{Q}}$.

- Let $p \geq 5$ be a prime and let $\operatorname{Cl}\left(\mathbb{Q}\left(\mu_{p}\right)\right)$ denote the class group of $\mathbb{Q}\left(\mu_{p}\right)$.

- The $\mathbb{F}_{p}\left[G_{\mathbb{Q}}\right]$-module $\mathcal{C}:=\mathrm{Cl}\left(\mathbb{Q}\left(\mu_{p}\right)\right) \otimes \mathbb{F}_{p}$ decomposes into a direct sum of isotypiccomponents $\mathcal{C}=\bigoplus_{i=0}^{p-2} \mathcal{C}\left(\bar{\chi}^{i}\right)$.

- We assume that there exists an odd integer $i \neq(p-1) / 2$ for which $2 \leq i \leq p-3$ and such that the isotypic component $\mathcal{C}\left(\bar{\chi}^{i}\right) \neq 0$. The adjoint Galois module associated with $\bar{\rho}$ of Theorem 1.1 by $A d^{0} \bar{\rho}$. It consists of matrices $\left(\begin{array}{cc}a & b \\ c & -a\end{array}\right)$ of trace zero over $\mathbb{F}_{p}$ on which $g \in G_{\mathbb{Q}}$ acts via conjugation by $\bar{\rho}(g)$.

- Let $\mathfrak{e}$ be the matrix $\mathfrak{e}=\left(\begin{array}{cc}1 & 0 \\ 0 & -1\end{array}\right)$. The subspace consisting of diagonal matrices is denoted by $\operatorname{Diag}(\bar{\rho}):=\mathbb{F}_{p} \mathfrak{e}$. 
- The ring of dual numbers $\mathbb{F}_{p}[\epsilon]:=\mathbb{F}_{p}[T] /\left(T^{2}\right)$ and $\epsilon$ denotes $T \bmod T^{2}$.

- Let $\mathcal{C}_{\mathbb{Z}_{p}}$ be the category of coefficient rings over $\mathbb{Z}_{p}$. The objects of $\mathcal{C}_{\mathbb{Z}_{p}}$ consist of Noetherian local $\mathbb{Z}_{p}$-algebras $R$ with maximal ideal $\mathfrak{m}$ endowed with an isomorphism

$$
\phi: R / \mathfrak{m} \stackrel{\sim}{\rightarrow} \mathbb{F}_{p} .
$$

A morphism of coefficient rings is a homomorphism of $\mathbb{Z}_{p}$-algebras compatible with residual isomorphisms.

\section{Lifting to Characteristic Zero}

We proceed to describe the local deformation condition at $p$ which will in particular ensure that our deformations are unramified over $\mathbb{Q}\left(\mu_{p^{\infty}}\right)$ at all primes $\mathfrak{p} \mid p$.

Definition 3.1. Let $\mathcal{F}_{p}: \mathcal{C}_{\mathbb{Z}_{p}} \rightarrow$ Sets be the functor of deformations of $\bar{\rho}$ which consist of a sum of two characters, we refer deformations $\mathcal{F}_{p}$ as diagonal. In greater detail, $\mathcal{F}_{p}(R)$ consists of deformations $\rho_{R}: G_{p} \rightarrow \mathrm{GL}_{2}(R)$ of $\bar{\rho}$ such that

- $\rho_{\left\lceil G_{p}\right.} \simeq \varphi_{1} \oplus \varphi_{2}$ where $\varphi_{1}$ and $\varphi_{2}$ are two characters (possibly ramified)

- $\operatorname{det} \rho=\chi^{i+p^{2}(p-1)}$.

The tangent space $\mathcal{N}_{p}$ is defined as the set of deformations of $\bar{\rho}$ to the dual numbers $\mathcal{F}_{p}\left(\mathbb{F}_{p}[\epsilon]\right)$ which has a natural structure of an $\mathbb{F}_{p}$ vector space and is realized as a subspace of $H^{1}\left(G_{p}, A d^{0} \bar{\rho}\right)$.

The functor $\mathcal{F}_{p}$ is a liftable deformation condition. Observe that since $G_{p}$ acts diagonally, the 1 dimensional space $\operatorname{Diag}\left(\bar{\rho}_{\left\lceil G_{p}\right.}\right)$ is a summand of $A d^{0} \bar{\rho}_{\left\lceil G_{p}\right.}$ and

$$
A d^{0} \bar{\rho}_{\left\lceil G_{p}\right.} \simeq \mathbb{F}_{p}\left(\bar{\chi}_{\left\lceil G_{p}\right.}^{i}\right) \oplus \operatorname{Diag}\left(\bar{\rho}_{\left\lceil G_{p}\right.}\right) \oplus \mathbb{F}_{p}\left(\bar{\chi}_{\left\lceil G_{p}\right.}^{-i}\right) .
$$

Definition 3.2. Let $\mathcal{F}$ be a deformation condition for $\bar{\rho}_{\mid G_{p}}$ with tangent space $\mathcal{N}_{\mathcal{F}}$.

- The deformation condition $\mathcal{F}$ is liftable if for every small extension $f: R \rightarrow S$, the induced map $f_{*}: \mathcal{F}(R) \rightarrow \mathcal{F}(S)$ is surjective.

- The deformation condition $\mathcal{F}$ is balanced if

$$
\operatorname{dim} \mathcal{N}_{p}=\operatorname{dim} H^{0}\left(G_{p}, \operatorname{Ad}^{0} \bar{\rho}\right)+1=2 .
$$

Proposition 3.3. The deformation condition $\mathcal{F}_{p}$ is liftable and balanced.

Proof. That $\mathcal{F}_{p}$ is liftable follows from Lemma 4.5 of [1]. We show that it is balanced, i.e. we deduce that

$$
\operatorname{dim} \mathcal{N}_{p}=\operatorname{dim} H^{0}\left(G_{p}, \operatorname{Ad}^{0} \bar{\rho}\right)+1=2 .
$$

Explicitly, $\mathcal{N}_{p}$ is the set of elements $X \in H^{1}\left(G_{p}, A d^{0} \bar{\rho}\right)$ for which the twist $\bar{\rho}(I d+\epsilon X)$ is diagonal, we deduce that $\mathcal{N}_{p}=H^{1}\left(G_{p}\right.$, $\left.\operatorname{Diag}(\bar{\rho})\right)$.

Since $\bar{\chi}_{\uparrow G_{p}}^{i} \neq 1$,

$$
\begin{aligned}
& \operatorname{dim} H^{0}\left(G_{p}, A d^{0} \bar{\rho}\right) \\
& =\operatorname{dim} H^{0}\left(G_{p}, \mathbb{F}_{p}\left(\bar{\chi}^{i}\right)\right)+\operatorname{dim} H^{0}\left(G_{p}, \mathbb{F}_{p}\right)+\operatorname{dim} H^{0}\left(G_{p}, \mathbb{F}_{p}\left(\bar{\chi}^{-i}\right)\right) \\
& =1 .
\end{aligned}
$$

We employ the Euler characteristic formula and local duality to compute $\operatorname{dim} \mathcal{N}_{p}$

$$
\begin{aligned}
\operatorname{dim} \mathcal{N}_{p} & =\operatorname{dim} H^{1}\left(G_{p}, \operatorname{Diag}(\bar{\rho})\right) \\
& =1+\operatorname{dim} H^{0}\left(G_{p}, \operatorname{Diag}(\bar{\rho})\right)+\operatorname{dim} H^{2}\left(G_{p}, \operatorname{Diag}(\bar{\rho})\right) \\
& =1+\operatorname{dim} H^{0}\left(G_{p}, \mathbb{F}_{p}\right)+\operatorname{dim} H^{0}\left(G_{p}, \mathbb{F}_{p}(\bar{\chi})\right) \\
& =\operatorname{dim} H^{0}\left(G_{p}, A d^{0} \bar{\rho}\right)+1=2 .
\end{aligned}
$$


The assumptions on $\bar{\rho}$ satisfy those on the assumptions laid out on the residual representations examined in 3 .

Lemma 3.4. The conditions stipulated in [3, Theorem 2] are satisfied by the representation $\bar{\rho}$.

Proof. The reducible representation $\bar{\rho}=\left(\begin{array}{ll}\phi & * \\ 0 & 1\end{array}\right)$ with $\phi=\bar{\chi}^{i}$, we recall that $i \neq(p-1) / 2$ is odd and $2 \leq i \leq p-3$. We enumerate the six conditions and show that they are satisfied:

- Condition (0) is satisfied since $p \neq 2$.

- Condition (1) requires that $\bar{\rho}$ is indecomposable (or in other words, not semi-simple), this follows by construction as the extension $E / \mathbb{Q}\left(\mu_{p}\right)$ is a non-trivial extension.

- Condition (2) requires that $\phi^{2} \neq 1$, or equivalently, $2 i \not \equiv 0 \bmod (p-1)$ which is satisfied.

- Condition (3) requires that $\phi \neq \bar{\chi}^{ \pm 1}$ which follows from the assumption on $i$.

- Condition (4) is automatically satisfied since the field in question $\mathbb{F}_{q}$ is $\mathbb{F}_{p}$.

- Since $i$ is odd, $\bar{\rho}$ is odd. Condition (5) requires that $\bar{\rho}$ that $\bar{\rho}_{\Gamma G_{p}}$ is not unramified of the form $\left(\begin{array}{ll}1 & * \\ 0 & 1\end{array}\right)$ (where $*$ may be trivial). The integer $i$ is divisible by $p-1$ and as a consequence, this condition is satisfied.

In the proof of Theorem 1.1 the following Lemma is applied in deducing that the image of a suitably constructed lift $\rho$ of $\bar{\rho}$ contains the principal congruence subgroup of $\mathrm{SL}_{2}\left(\mathbb{Z}_{p}\right)$.

Lemma 3.5. Let $p \geq 5$ be a prime, $X$ be a closed subgroup of $S L_{2}\left(\mathbb{Z}_{p}\right)$ and let $X_{2}$ be the image of $X$ in $S L_{2}\left(\mathbb{Z} / p^{2} \mathbb{Z}\right)$. Suppose that $X_{2}$ contains the principal congruence subgroup of $S L_{2}\left(\mathbb{Z} / p^{2} \mathbb{Z}\right)$, then $X$ contains the principal congruence subgroup of $S L_{2}\left(\mathbb{Z}_{p}\right)$.

Proof. The proof follows from that of Lemma 3 in [8. Chapter 4, Section 3.4] with very little modification.

Proof. (of Theorem 1.1 )

This result shall follow from the main result of [3] after a single modification is made to their construction. Their method relies on the existence of a balanced liftable deformation condition at each prime at which the residual Galois representation is allowed to ramify. There are cohomological obstructions to lifting a residual Galois representation which satisfies these local conditions. More specifically if a certain Selmer group does vanish the local to global deformation theoretic construction can be applied. On adjoining some auxiliary deformation conditions at a finite set of trivial primes (cf. Definition 12 in 3]) the associated Selmer group can be shown to vanish (cf. Proposition 46 in [3). Hamblen and Ramakrishna work throughout with the ordinary deformation condition (cf. [5] and [7]) at $p$. Instead, we shall prescribe the diagonal deformation condition which is also a balanced and liftable deformation. This was established in Proposition 3.3. The construction of Hamblen and Ramakrishna does not in any specific way utilize the ordinary deformation condition and the diagonal deformation condition may as well be used in its place since this condition is also liftable and balanced. It follows that there are infinitely many characteristic zero deformations $\rho$ satisfying the conditions of the main theorem.

In greater detail, it is a consequence of Proposition 42 of [3] that

$$
\text { image }\left\{\rho\left(G_{\mathbb{Q}\left(\mu_{p} \infty\right)}\right) \rightarrow \mathrm{SL}_{2}\left(\mathbb{Z} / p^{2} \mathbb{Z}\right)\right\}
$$

contains the principal congruence subgroup. It follows from Lemma 3.5 that $\rho\left(G_{\mathbb{Q}\left(\mu_{p} \infty\right)}\right)$ contains the principal congruence subgroup in $\mathrm{SL}_{2}\left(\mathbb{Z}_{p}\right)$. 
Proof. (of Corollory 1.2)

Let $\rho$ be a lift of $\bar{\rho}$ satisfying the conditions of Theorem 1.2. We let $L$ be the fixed field of $\rho$. The infinite cyclotomic field $\mathbb{Q}\left(\mu_{p^{\infty}}\right)$ is the fixed field of $\operatorname{det} \rho=\chi^{i+p^{2}(p-1)}$. Since $\rho\left(G_{\mathbb{Q}\left(\mu_{p} \infty\right)}\right)$ contains the principal congruence subgroup of $\mathrm{SL}_{2}\left(\mathbb{Z}_{p}\right)$ we see that $\operatorname{Gal}\left(L / \mathbb{Q}\left(\mu_{p^{\infty}}\right)\right)$ is topologically isomorphic to a subgroup of $\mathrm{SL}_{2}\left(\mathbb{Z}_{p}\right)$ which contains the principal congruence subgroup.

The local representation $\rho_{\left\lceil G_{p}\right.}$ is a sum of characters $\varphi_{1}$ and $\varphi_{2}$, we deduce that $L$ is unramified at all primes above $p$.

Since $\bar{\rho}_{\mid G_{\mathbb{Q}\left(\mu_{p}\right)}}$ is unramified at all primes, it follows that at the primes at which $L$ is ramified, $L$ must be tamely ramified.

\section{Proof. (of Proposition 1.3)}

Indeed if $p$ is such a prime, since $p$ is irregular, the quotient $\mathcal{C}=\mathrm{Cl}\left(\mathbb{Q}\left(\mu_{p}\right)\right) \otimes \mathbb{F}_{p} \neq 0$. By assumption each even eigenspace $\mathcal{C}\left(\bar{\chi}^{2 j}\right)=0$. Thus there exists an odd integer $1 \leq i \leq p-2$ for which $\mathcal{C}\left(\bar{\chi}^{i}\right) \neq 0$. Since $p \equiv 1 \bmod 4$, and $i$ is odd, we have that $i \neq \frac{p-1}{2}$. On the other hand, $i \neq 1$ since $\mathcal{C}(\bar{\chi})=0$ (cf. Proposition 6.16 of $[9]$ ). By Herbrand's theorem implies that $p$ divides the numerator of the Bernoulli number $B_{p-i}$. Since $B_{2}=\frac{1}{6}$ we deduce that $i \neq p-2$ and thus lies in the range $2 \leq i \leq p-3$.

\section{References}

[1] Blondeau, Julien. "Lifting of nearly extraordinary Galois representations." Journal of Number Theory 133.6 (2013) 2066-2082. https://doi.org/10.1016/j.jnt.2012.08.026

[2] Fontaine, Jean-Marc, and Barry Mazur. "Geometric Galois representations." Elliptic curves, modular forms, and Fermat's last theorem (Hong Kong, 1993), Ser. Number Theory, I (1995) 41-78.

[3] Hamblen, Spencer and Ramakrishna, Ravi. "Deformations of certain reducible Galois representations II". In:Amer. J. Math.130.4(2008) 913-944. https://doi.org/10.1353/ajm.0.0008

[4] Mazur, Barry. "Deforming galois representations." Galois Groups over $\mathbb{Q}$. Springer, New York, NY (1989) 385-437. https://doi.org/10.1007/978-1-4613-9649-9_7

[5] Ramakrishna, Ravi. "Deforming Galois representations and the conjectures of Serre and Fontaine-Mazur." Annals of mathematics 156.1 (2002) 115-154, https://doi.org/10.2307/3597186

[6] Ohtani, Sachiko. "Deformations of locally abelian Galois representations and unramified extensions." Journal of Number Theory 120.2 (2006) 272-286, https://doi.org/10.1016/j.jnt.2005.12.002

[7] Taylor, Richard. "On icosahedral Artin representations, II." American journal of mathematics 125.3 (2003) 549-566, http://muse.jhu.edu/journals/american_journal_of_mathematics/v125/125.3taylor.pdf

[8] Serre, Jean-Pierre. Abelian 1-adic representations and elliptic curves. AK Peters/CRC Press (1997).

[9] Washington, Lawrence C. Introduction to cyclotomic fields. Vol. 83. Springer Science and Business Media (1997). https://doi.org/10.1007/978-1-4612-1934-7.

Cornell University, Department of Mathematics, Malott Hall, Ithaca, NY 14853-4201 USA

E-mail address: ar2222@cornell.edu 